\title{
Editorial
}

\section{Analysis and Synthesis of Stochastic Nonlinear Systems}

\author{
Shouming Zhong, ${ }^{1}$ Jun Cheng, ${ }^{2}$ Jiuwen Cao, ${ }^{3}$ P. Balasubramaniam, ${ }^{4}$ and Haibo $\mathrm{Du}^{5}$ \\ ${ }^{1}$ School of Mathematical Sciences, University of Electronic Science and Technology of China, Chengdu 611731, China \\ ${ }^{2}$ School of Science, Hubei University for Nationalities, Hubei 445000, China \\ ${ }^{3}$ Key Lab for IOT and Information Fusion Technology of Zhejiang, Hangzhou Dianzi University, Zhejiang 310038, China \\ ${ }^{4}$ Department of Mathematics, Gandhigram Rural University, Gandhigram 624302, India \\ ${ }^{5}$ Department of Electrical and Computer Engineering, University of Texas at San Antonio, San Antonio, TX 78249, USA
}

Correspondence should be addressed to Shouming Zhong; zhongsm@uestc.edu.cn

Received 7 July 2015; Accepted 7 July 2015

Copyright (C) 2015 Shouming Zhong et al. This is an open access article distributed under the Creative Commons Attribution License, which permits unrestricted use, distribution, and reproduction in any medium, provided the original work is properly cited.

Ever since the emergence of stochastic stabilization theory in the 1960s, stochastic systems have been widely studied and introduced in the areas of mathematics and engineering over the past few years. It should be noted that the stochastic dynamical system is a dynamical system subjected to the effects of noise, and the effects of fluctuations have been receiving increasing interest. The stochastic abrupt changes, such as sudden environment changes and repairs of components and random failures, always exist in the variable structures during the stochastic dynamical systems; the nonlinearity occurs in the real engineering applications. Nowadays, researches on stochastic nonlinear systems have received significant attention due to their wide applications in various fields such as physics, technology, and life sciences. Therefore, the analysis and synthesis of stochastic nonlinear systems play important roles in many practical systems.

In the light of above considerations, this special issue has been launched. Up to now, papers were submitted by researchers in various research fields from different countries, and a total number of papers are included in this special issue. The accepted reviewed papers can be categorized into five groups: nonlinear Markovian jump systems, neural networks with external constant input vector, noise included nonlinear oscillator, Brownian Motions, and other nonlinear character applications.

In nonlinear Markovian jump systems, $\mathrm{H}$. Dong and J. Chen introduced concerned with the $H_{\infty}$ filtering for a class of networked Markovian jump systems with multiple communication delays. The stochastic communication delays are considered in the filter design. L. Li et al. investigated
$H_{\infty}$ fuzzy control for a class of nonlinear singular Markovian jump systems where time delay under consideration is described by Takagi-Sugeno (T-S) fuzzy models. B. Yan et al. considered the full information of underlying Markov process; $H_{\infty}$ filtering is designed for underlying closed-loop singular Markovian jump system while system state does not exceed a given bound over some finite time interval.

In neural networks with external constant input vector, with a suitable Lyapunov-Krasovskii functional (LKF) and Writinger-based integral inequality, $M$. J. Park proposed the sufficient conditions for guaranteeing the asymptotic stability of the concerned networks which are derived in terms of linear matrix inequalities. J. Tian and Y. Liu established some less conservative stability criteria in terms of linear matrix inequalities (LMIs) by constructing a new LyapunovKrasovskii functional (LKF) in each subinterval and using the reciprocally convex approach. W. Wen and K. Shi proposed some new less conservative conditions by taking fully the relationship between the terms in the Leibniz-Newton formula and some effective mathematical techniques and a convex optimization approach into account. L. Hou and $\mathrm{H}$. Zhu investigated the stability of stochastic discrete-time neural networks (NNs) with discrete time-varying delays and leakage delay by constructing a novel Lyapunov-Krasovskii function based on stability theory.

In a noise included nonlinear oscillator, G. Ge and Z. C. Yun studied the deterministic and the stochastic responses by applying the improved complex normal form method based on the He's energy balance method. Q. Gao et al. proposed simplified theoretical derivations to overcome the defects in 
which most of studies focused on single-factor analysis or multifactor analysis based on full factorial design. B. Wang and X. Dong proposed two kinds of chaotic secure communication schemes in the case of existence of system disturbances and possible real-world applications.

In Brownian motions, J. Miao and X. Yang obtained the existence and uniqueness of the solutions to BSDEs driven by multidimensional fractional Brownian motions, where a fixed point principle is employed. L. Liu and C. Liu presented a novel nonlinear four-dimensional hyperchaotic system and its fractional-order form. G. Qin and J. Zou proposed the randomness of delay and a rapid response, an $H_{\infty}$-based delaytolerant linear quadratic regulator (LQR) control method based on Taylor series expansion.

In other nonlinear character applications, M. Hui et al. proposed a new Volterra prediction method based on phase space reconstruction theory and Lyapunov exponent to analyze the nonlinear character of traffic flow. Y. Zhou et al. obtained parameter bifurcation sets that divide the parameter space into different regions which correspond to qualitatively different phase portraits. Y. Wang et al. considered the positivity and boundedness of the solution and the existence, local stability, and global stability of equilibria. A. Wang et al. introduced the CEV model and its application in a study of optimal investment strategy. Y. Tang et al. presented a novel surveillance system named Thermal Omnidirectional Vision (TOV) system which can work in total darkness with a wild field of view. J. Meng et al. explored a new deflated BiCG method and an application to model reduction. D. Luo and A. Liu constructed a Kernel Fisher discriminant analysis (KFDA) method from well logs for lithology identification purposes. J. Ma et al. investigated the hysteretic behavior of concrete-filled steel tube arch by using a fiber beam element. Z. Cui et al. proposed a hierarchical recognition system (HRS) based on constrained deep belief network (DBN) for SAR automatic target recognition (SAR ATR). Z.-Y. Xiang et al. proposed a new partitioning method, called Wedging Insertion for solving large scale classical symmetric Traveling Salesman Problem (TSP).

\title{
Acknowledgments
}

As the guest editors of the special issue, we would like to express our gratitude to all the authors who have contributed their work to our special issue. We also would like to express our sincere appreciation to the reviewers for their valuable and insightful comments on the submitted manuscripts to our special issue.

\author{
Shouming Zhong \\ Jun Cheng \\ Jiuwen Cao \\ P. Balasubramaniam \\ Haibo Du
}




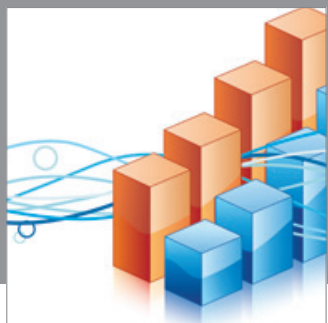

Advances in

Operations Research

mansans

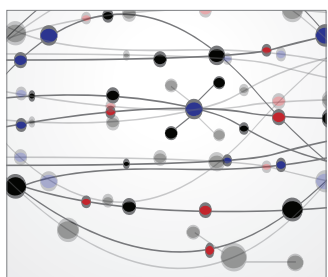

The Scientific World Journal
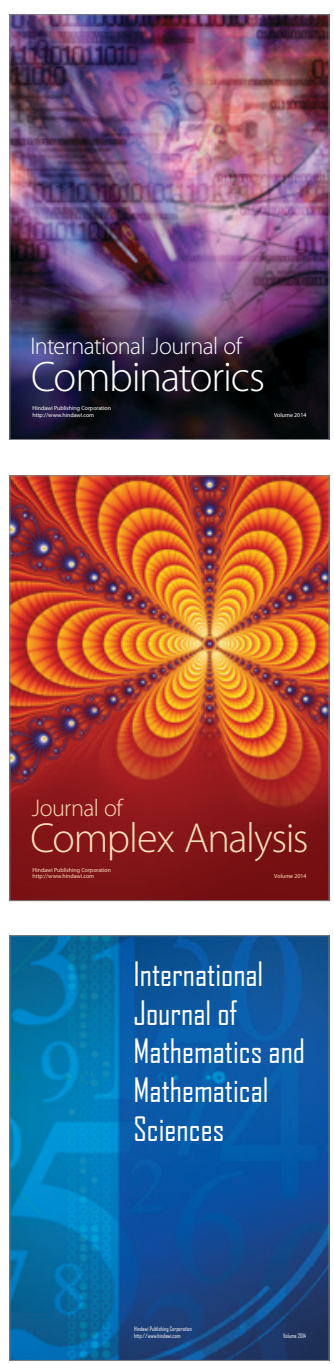
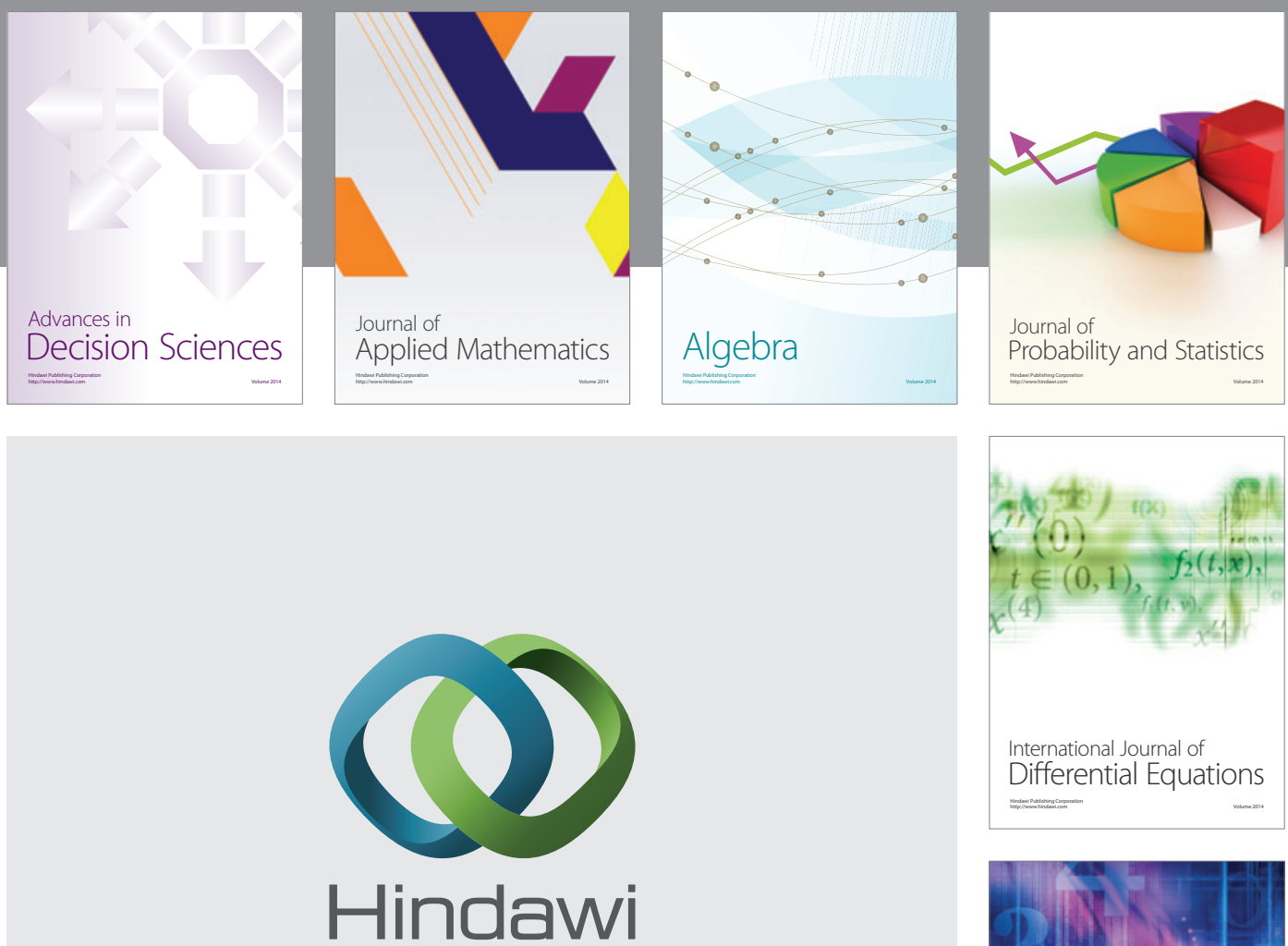

Submit your manuscripts at http://www.hindawi.com
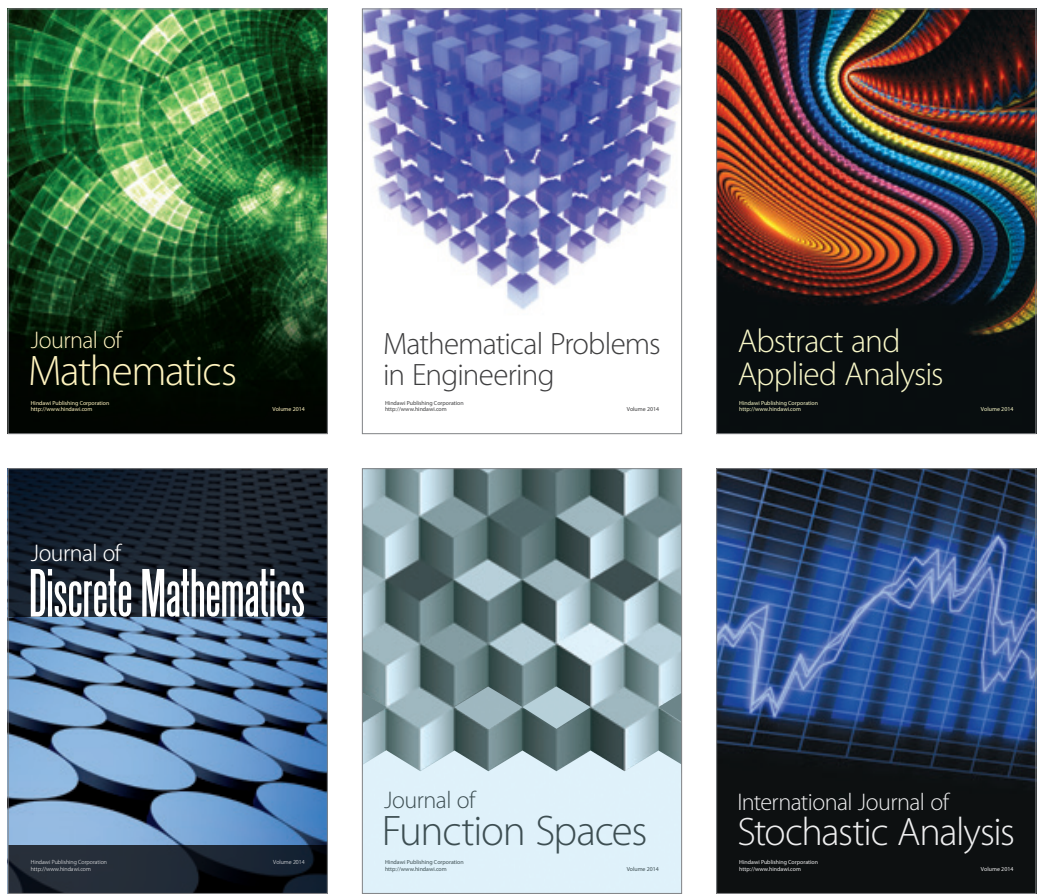

Journal of

Function Spaces

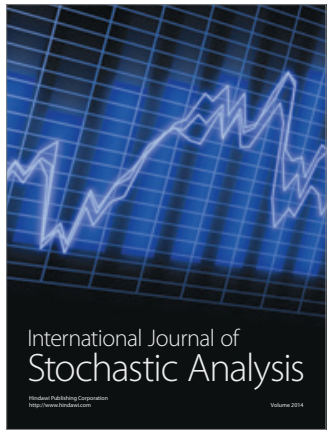

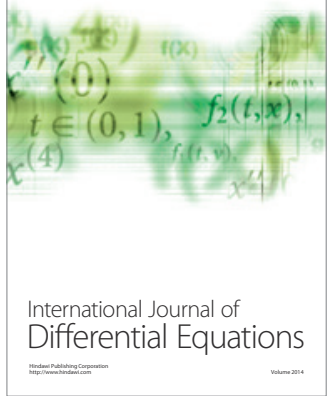
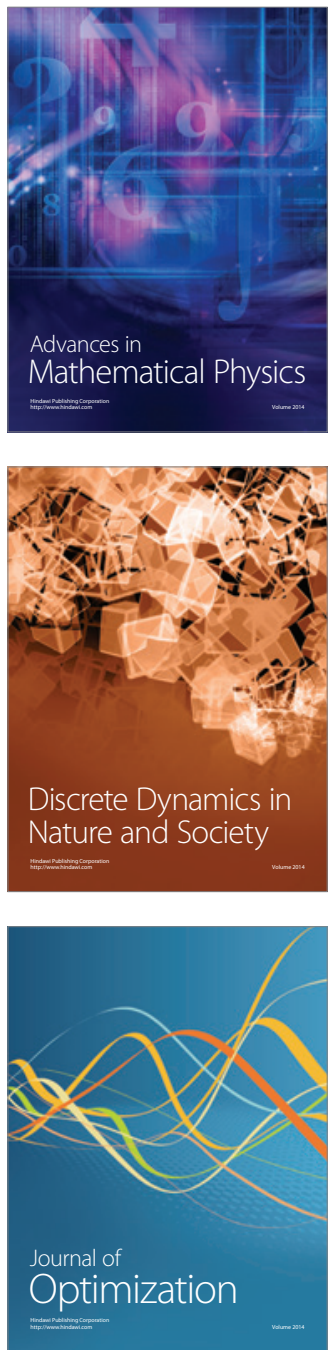